\title{
THE COSMIC MICROWAVE BACKGROUND BISPECTRUM AS A TEST OF THE PHYSICS OF INFLATION AND PROBE OF THE ASTROPHYSICS OF THE LOW-REDSHIFT UNIVERSE
}

\author{
EIICHIRO KOMATSU ${ }^{1,2,3}$ AND DAVID N. SPERGEL ${ }^{1,4}$ \\ ${ }^{1}$ Department of Astrophysical Sciences, Princeton University, Princeton, NJ 08544 , \\ USA \\ ${ }^{2}$ School of Natural Sciences, Institute for Advanced Study, Princeton, NJ 08540, USA \\ ${ }^{3}$ Astronomical Institute, Tôhoku University, Aoba, Sendai 980-8578, Japan \\ ${ }^{4}$ Keck Distinguished Visiting Professor, School of Natural Sciences, Institute for \\ Advanced Study, Princeton, NJ 08540, USA \\ E-mail: komatsu@astro.princeton.edu,dns@astro.princeton.edu
}

\begin{abstract}
Why is non-Gaussianity interesting? One of generic predictions from inflationary scenarios is that primordial fluctuations are exactly Gaussian in linear order; however, the non-linearity in the inflation will produce weak non-Gaussianity. Thus, measuring the non-Gaussianity in the cosmic microwave background radiation anisotropy is a probe of the non-linear physics in the very early universe. Since the angular three point function is zero for the Gaussian field, it is sensitive to the non-Gaussianity. We predict its harmonic transform counterpart, the angular Bispectrum, down to arcminutes angular scales, including the full effect of the radiation transfer function. We find that even the Planck experiment cannot detect the primary bispectrum from the inflation, as long as the single field slowroll inflation is right. Non-linearities in the low redshift universe also produce the non-Gaussianity. We find that secondary bispectra are detectable by both MAP and Planck experiments. The secondary bispectra probe the non-linear physics of the low-redshift universe. Although this could be a contaminant to the primary signal, MAP and Planck experiments are found to be able to separate the primary from secondary effects well. We present a tentative comparison of the primary bispectrum to the published COBE 4 year bispectrum. The data put a weak constraint on the parameter, and the constraint would become much tighter when we use all modes available in the COBE data, and certainly forthcoming satellite experiments. As a conclusion, the bispectrum is a key measure to confirm or destroy the simple inflationary scenario in non-linear order that seems quite successful in linear order.
\end{abstract}

\section{The CMB non-Gaussianity from inflation}

\subsection{Order-of-magnitude estimation of the non-linear coupling parameter $f_{N L}$}

We briefly consider the mechanism producing the primary CMB non-Gaussianity from the inflation. Since the observed CMB anisotropy $\Delta T / T$ is thought to be generated by curvature perturbations $\Phi$, the primary non-Gaussianity should be encapsulated in $\Phi$. The inflation makes $\Phi$ weakly non-Gaussian. However, even if $\Phi$ is Gaussian, $\Delta T / T$ can be non-Gaussian.

$\Delta T / T$ is related to $\Phi$ through the non-linear relation:

$$
\frac{\Delta T}{T} \sim g_{T}\left(\Phi+f_{\Phi} \Phi^{2}\right),
$$

where $g_{T}$ is the radiation transfer function converting $\Phi$ to $\Delta T / T . g_{T}=-1 / 3$ for the Sachs-Wolfe effect 1 . The second term with $f_{\Phi} \sim \mathcal{O}(1)$ is the higher order correction that arises from the second order gravitational perturbation theory2. Thus,

bispectrum 'mg9'long: submitted to World Scientific on October 24, 2018 
it makes $\Delta T$ weakly non-Gaussian, even when $\Phi$ is Gaussian. $\Phi$ is actually a weakly non-Gaussian field, and thought to be produced from the inflaton fluctuations $\delta \phi$ through the relation 3 :

$$
\Phi \sim m_{p l}^{-1} g_{\Phi}\left(\delta \phi+m_{p l}^{-1} f_{\delta \phi} \delta \phi^{2}\right) .
$$

$g_{\Phi} \sim \mathcal{O}(10)$, and $f_{\delta \phi} \sim \mathcal{O}\left(10^{-1}\right)$ for a class of slow-roll inflationary scenarios with the single inflaton field. If the dynamics of $\delta \phi$ field is simple enough to keep itself Gaussian throughout the evolution, then we can stop our consideration here. However, in generic, the non-trivial form of the interaction term in the equation of motion 5 , or the non-linear coupling between the coarse-grained classical fluctuations and the fine scale quantum fluctuation 4 , 6 , make $\delta \phi$ weakly non-Gaussian, even if it is originally Gaussian field. The result is simply written as

$$
\delta \phi \sim g_{\delta \phi}\left(\eta+m_{p l}^{-1} f_{\eta} \eta^{2}\right),
$$

where $\eta$ is Gaussian, $g_{\delta \phi} \sim 1$, and $f_{\eta} \sim \mathcal{O}\left(10^{-1}\right)$.

It is then useful to rewritten equations (11)-(3) as

$$
\frac{\Delta T}{T} \sim g_{T}\left[\Phi_{L}+\left(f_{\Phi}+g_{\Phi}^{-1} f_{\delta \phi}+g_{\Phi}^{-1} g_{\delta \phi}^{-1} f_{\eta}\right) \Phi_{L}^{2}\right],
$$

where $\Phi_{L} \equiv g_{\Phi} g_{\delta \phi} \eta \sim 10 \eta$ is the Gaussian part of curvature perturbations. More specifically, we define the non-linear coupling parameter, $f_{N L}$, as

$$
\Phi(\mathbf{x})=\Phi_{L}(\mathbf{x})+f_{N L}\left(\Phi_{L}^{2}(\mathbf{x})-\left\langle\Phi_{L}^{2}(\mathbf{x})\right\rangle\right),
$$

where the angular bracket denotes the statistical ensemble average. Note that $f_{N L} \sim f_{\Phi}+g_{\Phi}^{-1} f_{\delta \phi}+g_{\Phi}^{-1} g_{\delta \phi}^{-1} f_{\eta}$, and the first term $\sim \mathcal{O}(1)$ from the second order gravity effect is dominant compared to other two terms from slow-roll inflation $\sim \mathcal{O}\left(10^{-2}\right) . f_{N L}$ corresponds to $\alpha_{\Phi}$ in Verde et al.. .

\subsection{The reduced angular bispectrum}

We use the angular bispectrum, harmonic transform of the angular three point function, as a measure of the CMB non-Gaussianity.

$\Delta T(\hat{\mathbf{n}}) / T$ in the sky is expanded into the spherical harmonics: $\Delta T(\hat{\mathbf{n}}) / T=$ $\sum_{l m} a_{l m} Y_{l m}(\hat{\mathbf{n}}) . a_{l m}$ is then written in terms of $\Phi$-field,

$$
a_{l m}=4 \pi(-i)^{l} \int \frac{d^{3} \mathbf{k}}{(2 \pi)^{3}} \Phi(\mathbf{k}) g_{T l}(k) Y_{l m}^{*}(\hat{\mathbf{k}}) .
$$

$\Phi(\mathbf{k})$ is weakly non-Gaussian field given by the Fourier transform of equation (5). Instead of assuming the Sachs-Wolfe's $-1 / 3$ law for $g_{T l}(k)$, we use the full $g_{T l}(k)$ down to small scales by solving the Boltzmann transport equation with the CMBFAST codel. We then define the reduced angular bispectrum $b_{l_{1} l_{2} l_{3}}$ as

$$
\begin{aligned}
b_{l_{1} l_{2} l_{3}} \equiv & \sqrt{\frac{4 \pi}{\left(2 l_{1}+1\right)\left(2 l_{2}+1\right)\left(2 l_{3}+1\right)}}\left(\begin{array}{ccc}
l_{1} & l_{2} & l_{3} \\
0 & 0 & 0
\end{array}\right)^{-1} \\
& \times \sum_{m_{1} m_{2} m_{3}}\left(\begin{array}{ccc}
l_{1} & l_{2} & l_{3} \\
m_{1} & m_{2} & m_{3}
\end{array}\right)\left\langle a_{l_{1} m_{1}} a_{l_{2} m_{2}} a_{l_{3} m_{3}}\right\rangle,
\end{aligned}
$$

bispectrum 'mg9'long: submitted to World Scientific on October 24, 2018 


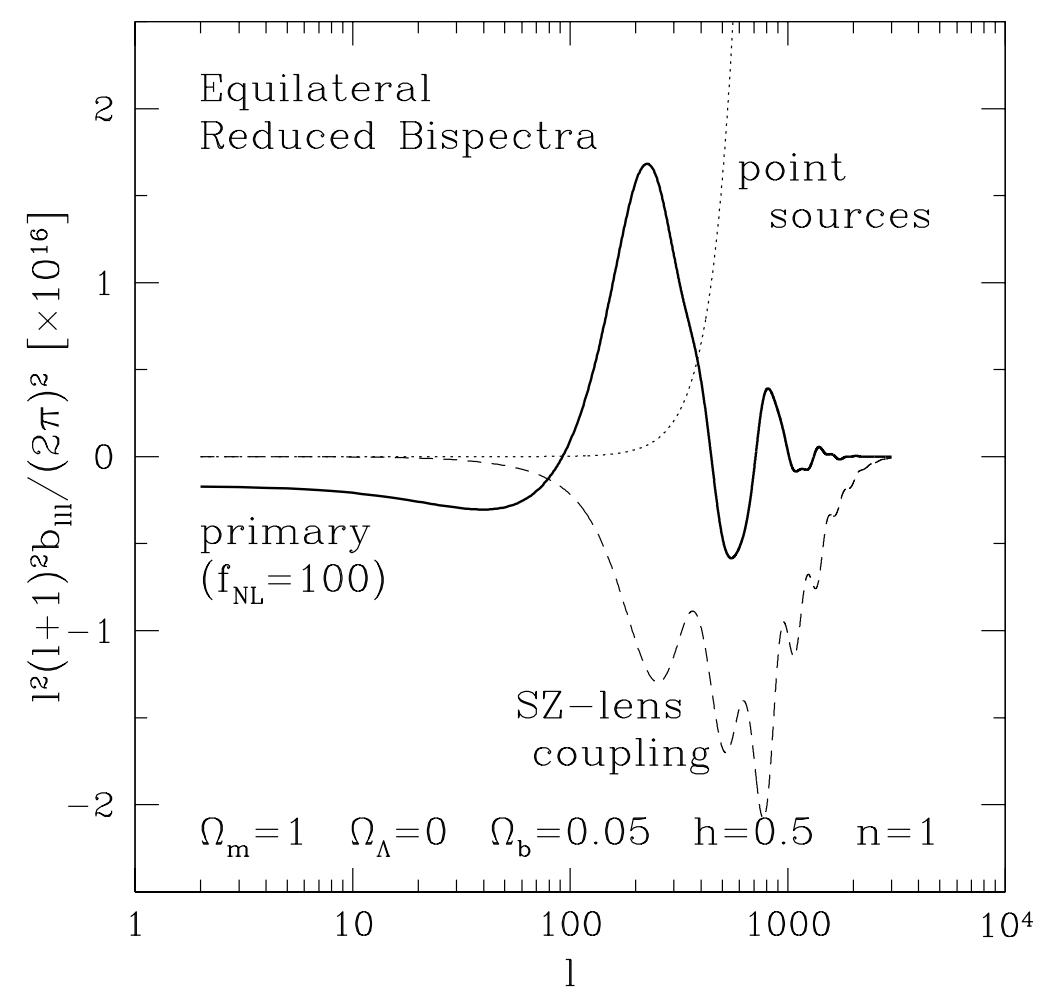

Figure 1. The equilateral configuration of the reduced bispectra, $\left[l^{2}(l+1)^{2} b_{l l l} /(2 \pi)^{2}\right] \times 10^{16}$. The weight is motivated by the behavior of the primary component, $b_{i l l}^{\text {primary }} \propto l^{-4}$. Solid line shows the primary bispectrum from inflation with $f_{N L}=100$ (see eq. (5i)). Dashed line shows the Sunyaev-Zel'dovich effect and weak lensing coupling in the Rayleigh-Jeans limit, while dotted line shows the extragalactic radio and infrared point sources.

where the matrix is the Wigner- $3 j$ symbol. $b_{l_{1} l_{2} l_{3}}$ is found to be more useful to describe the physical properties of the angular bispectrum than the conventional angular averaged bispectrum, $B_{l_{1} l_{2} l_{3}} \equiv \sum_{m_{1} m_{2} m_{3}}\left(\begin{array}{ccc}l_{1} & l_{2} & l_{3} \\ m_{1} & m_{2} & m_{3}\end{array}\right)\left\langle a_{l_{1} m_{1}} a_{l_{2} m_{2}} a_{l_{3} m_{3}}\right\rangle$. Note that Magueijo 10 defines a similar quantity, $\hat{B}_{l_{1} l_{2} l_{3}}=b_{l_{1} l_{2} l_{3}} / \sqrt{4 \pi}$.

We give the explicit form of the primary reduced bispectrum in the referencel. Figure 1 plots the equilateral case $\left(l_{1}=l_{2}=l_{3} \equiv l\right)$ reduced bispectrum. The acoustic oscillation in $g_{T l}(k)$ appears in $b_{l l l}$. For the scale invariant $\Phi_{L}$-field power spectrum,

$$
b_{l l l}^{\text {primary }} \sim 10^{-17} f_{N L} \times l^{-4},
$$

apart from the oscillation and the Silk damping. 


\section{The CMB non-Gaussianity from low-redshift universe}

Secondary bispectra from the coupling between the Sunyaev-Zel'dovich (SZ) effect and the weak lensing effect 11.12 , and the extragalactic radio and infrared sources 13 are found to be marginally detectable by MAP, while Planck will detect them significantly.

We give the explicit form of the SZ-lensing coupling bispectrum in the reference $\mathrm{g}$. It is roughly

$$
b_{l l l}^{s z-l e n s} \sim 10^{-19} j_{\nu} \bar{T}_{\rho 0} b_{\text {gas }} \times l^{-3},
$$

up to $l \sim 1000 . \bar{T}_{\rho 0} \sim 0.3$ is the present day density weightedmean temperature in units of $1 \mathrm{keV}$, while $b_{\text {gas }} \sim 6$ is the linear gas pressure bias 44 . $j_{\nu}$ is the spectral function of the SZ effect, and equals -2 in the Rayleigh-Jeans regime 5 .

The CMB bispectrum from extragalactic point sources is easy to estimate, as long as they are assumed to be Poisson distributed. The reduced bispectrum is just constant in that case, i.e., $b_{l_{1} l_{2} l_{3}}^{p s}=b^{p s}=$ constant. Detailed arguments are summarized in the referencel. The results are

$$
\begin{aligned}
b^{p s}(90 \mathrm{GHz},<2 \mathrm{Jy}) & \sim 2 \times 10^{-25}, \\
b^{p s}(217 \mathrm{GHz},<0.2 \mathrm{Jy}) & \sim 5 \times 10^{-28} .
\end{aligned}
$$

Equations (10) and (11) correspond to MAP and Planck experiments, respectively, after subtracting $>5 \sigma$ sources from the map.

Figure 1 plots $b_{l l l}^{s z-l e n s}$ in the Rayleigh-Jeans limit and $b^{p s}$. Clearly, secondary sources dominate the primary signal as going to smaller angular scales, even if $f_{N L}=100$ is taken. This is opposite to the CMB power spectrum that is dominated by either the primary CMB or the instrumental noise at all $l$ in the COBE, MAP, and Planck experiments. Thus, we need to separate the primary bispectrum from secondary bispectra. Fortunately, the shapes of bispectra are fairly different, so that we can discriminate among them based on shapes.

\section{Can we detect/separate the CMB bispectra?}

Since the CMB bispectrum is very weak compared to the Gaussian noise from CMB itself, it is totally hopeless to measure $b_{l_{1} l_{2} l_{3}}$ per mode. Instead, we fit the observed data by theoretical bispectra, and then constrain parameters. For this purpose, we minimize $\chi^{2}$ given by

$$
\chi^{2}\left(A_{i}\right) \equiv \sum_{2 \leq l_{1} \leq l_{2} \leq l_{3}} \frac{\left(B_{l_{1} l_{2} l_{3}}^{o b s}-\sum_{i} A_{i} B_{l_{1} l_{2} l_{3}}^{(i)}\right)^{2}}{\sigma_{l_{1} l_{2} l_{3}}^{2}},
$$

where $i$ denotes a component such as the primary, the SZ and lensing effects, extragalactic sources, and so on. In case that the non-Gaussianity is small, the cosmic variance of the bispectrum $\sigma_{l_{1} l_{2} l_{3}}^{2}$ is given by the six-point function of $a_{l m} 16$. 17 . $\sigma_{l_{1} l_{2} l_{3}}^{2}$ is dominated by either the primary CMB or the instrumental noise, while

bispectrum mg9'long: submitted to World Scientific on October 24, 2018 
Table 1. The sigpal-to-noise ratio for the detection of bispectra. $f_{N L}$ is the non-linear coupling parameter (Eq. (5)). $\bar{T}_{\rho 0} \sim 0.3$ is the present day density weighted mean temperature in units of $1 \mathrm{keV}$, while $b_{\text {gas }} \sim 6$ is the linear gas pressure bias. $j_{\nu}$ is the spectral function of the Sunyaev-Zel'dovich effect that has -2 in the Rayleigh-Jeans regime. $b_{25}^{p s} \equiv b^{p s} / 10^{-25}$, and $b_{27}^{p s} \equiv b^{p s} / 10^{-27}$, where $b^{p s}$ is the reduced Poisson bispectrum.

\begin{tabular}{c|ccc}
\hline & primary & SZ-lensing & point sources \\
\hline COBE & $1.7 \times 10^{-3} f_{N L}$ & $1.8 \times 10^{-4}\left|j_{\nu}\right| \bar{T}_{\rho 0} b_{\text {gas }}$ & $5.7 \times 10^{-7} b_{25}^{p s}$ \\
MAP & $5.8 \times 10^{-2} f_{N L}$ & $0.34\left|j_{\nu}\right| \bar{T}_{\rho 0} b_{\text {gas }}$ & $2.2 b_{25}^{\text {ps }}$ \\
Planck & $0.19 f_{N L}$ & $6.2\left|j_{\nu}\right| \bar{T}_{\rho 0} b_{\text {gas }}$ & $52 b_{27}^{\text {ps }}$ \\
\hline
\end{tabular}

Table 2. The signal degradation parameters. $d_{i}$ is defined so as $d_{i}=1$ for perfectly separated component, while $d_{i}>1$ for poorly separated one.

\begin{tabular}{c|ccc}
\hline & primary & SZ-lensing & point sources \\
\hline COBE & 1.46 & 3.89 & 3.45 \\
MAP & 1.01 & 1.16 & 1.14 \\
Planck & 1.00 & 1.00 & 1.00 \\
\hline
\end{tabular}

the secondary sources are subdominant as long as COBE, MAP, and Planck experiments are considered. Taking $\partial \chi^{2} / \partial A_{i}=0$, we obtain the Fisher matrix,

$$
F_{i j} \equiv \sum_{2 \leq l_{1} \leq l_{2} \leq l_{3}} \frac{B_{l_{1} l_{2} l_{3}}^{(i)} B_{l_{1} l_{2} l_{3}}^{(j)}}{\sigma_{l_{1} l_{2} l_{3}}^{2}} .
$$

Since the covariance matrix of $A_{i}$ is $F_{i j}^{-1}$, we define the signal-to-noise ratio $(S / N)_{i} \equiv 1 / \sqrt{F_{i i}^{-1}}$ for a component $i$, and the signal degradation parameter $d_{i} \equiv F_{i i} F_{i i}^{-1}$ due to a contamination from correlated components. $d_{i}$ is defined so as $d_{i}=1$ for perfectly separated component, while $d_{i}>1$ for poorly separated one. Table 1 and 2 summarize $(S / N)_{i}$ and $d_{i}$, respectively, for COBE, MAP, and Planck experiments. In order to obtain $(S / N)_{\text {primary }}>1, f_{N L}>600,20$, and 5 are needed for COBE, MAP, and Planck experiments. As derived in section 1.1, the single field slow-roll inflation generally predicts $f_{N L} \sim \mathcal{O}\left(10^{-2}\right)$, while the second order gravity yields $f_{N L} \sim \mathcal{O}(1)$. Therefore, either way, the detection is not expected for those experiments.

$(S / N)_{s z-l e n s} \sim 1$ and $(S / N)_{p s} \sim 4$ are expected for MAP, and $(S / N)_{s z-l e n s} \sim$ 20 and $(S / N)_{p s} \sim 30$ for Planck. Thus, the leading contribution to the CMB bispectrum would be from extragalactic point sources. Then, the SZ-lensing contribution would be measured by Planck, yielding a constraint on $\bar{T}_{\rho 0} b_{\text {gas }}$. This quantity measures the physical state of intergalactic gas pressure that is uncertain at present.

\section{Tentative comparison to the COBE 4 year bispectrum}

Ferreira, Magueijo, and Górski18 claimed a significant detection of the equilateral bispectrum at $l=16$ in the publicly available COBE 4 year data. Banday, Zaroubi,

bispectrum mg9 long: submitted to World Scientific on October 24, 2018 
and Górsk 19 found that their detection would be ascribed to the systematics associated with the eclipse of COBE satellite. This partly demonstrates that the bispectrum is sensitive to the non-linear effect even including the systematics in data.

Magueijo 10 tabulates a particular configuration of the COBE bispectrum without eclipse data. He tabulates 8 modes of

$$
J_{l}^{3} \equiv \frac{b_{l-1 l l+1}}{\sqrt{4 \pi C_{l-1} C_{l} C_{l+1}}}
$$

for $l=4-18$. Figure 2 compares our primary bispectrum to his data. There is no significant detection of $J_{l}^{3}$ per mode, and then $f_{N L}$ is constrained as $f_{N L}<10^{4}$. It should be stressed that only 8 modes were used to constrain $f_{N L}$ here. Since the total number of modes available in the full COBE bispectrum is 466 up to $l=20$, the constraint on $f_{N L}$ becomes much tighter than $<10^{4}$, say, $1 \sigma \sim 600$ as suggested by our estimation (table 1 ). MAP and Planck will certainly put a very stringent constraint on $f_{N L}$, and thus test the inflationary scenario in non-linear order.

\section{Discussion}

What if the primary bispectrum is detected? In other words, what if $f_{N L} \gg 1$ is measured? One might suspect the systematics, as already demonstrated in the COBE data, or foreground secondary sources. Since we fit the theory to the data, the goodness-of-fit value of $\chi^{2}$ at the best-fit $f_{N L}$ would tell us whether detected signal is really primary or not. If it passes the $\chi^{2}$ test, then the most straightforward consequence is that - the single field slow-roll inflation is not right.

\section{Acknowledgments}

We would like to thank Licia Verde for invaluable discussion. E. K. acknowledges a fellowship from the Japan Society for the Promotion of Science. D. N. S. is partially supported by the MAP/MIDEX program.

\section{References}

1. R. K. Sachs and A. M. Wolfe, Astrophys. J. 147, 73 (1967).

2. T. Pyne and S. M. Carroll, Phys. Rev. D 53, 2920 (1996).

3. D. S. Salopek and J. R. Bond, Phys. Rev. D 42, 3936 (1990); ibid. 43, 1005 (1991).

4. A. Gangui, F. Lucchin, S. Matarrese and S. Mollerach, Astrophys. J. 430, 447 (1994).

5. T. Falk, R. Rangarajan and M. Srednicki, Astrophys. J. Lett. 403, L1 (1993).

6. A. A. Starobinsky, in Field Theory, Quantum Gravity, and Strings, edited by H. T. de Vega and N. Sanchez, Lecture Notes in Physics, Vol. 246 (SpringerVerlag, Berlin, 1986), p.107.

7. L. Verde, L. Wang, A. F. Heavens and M. Kamionkowski, Mon. Not. R. Astron. Soc. 313, 141 (2000).

bispectrum mg9'long: submitted to World Scientific on October 24, 2018 


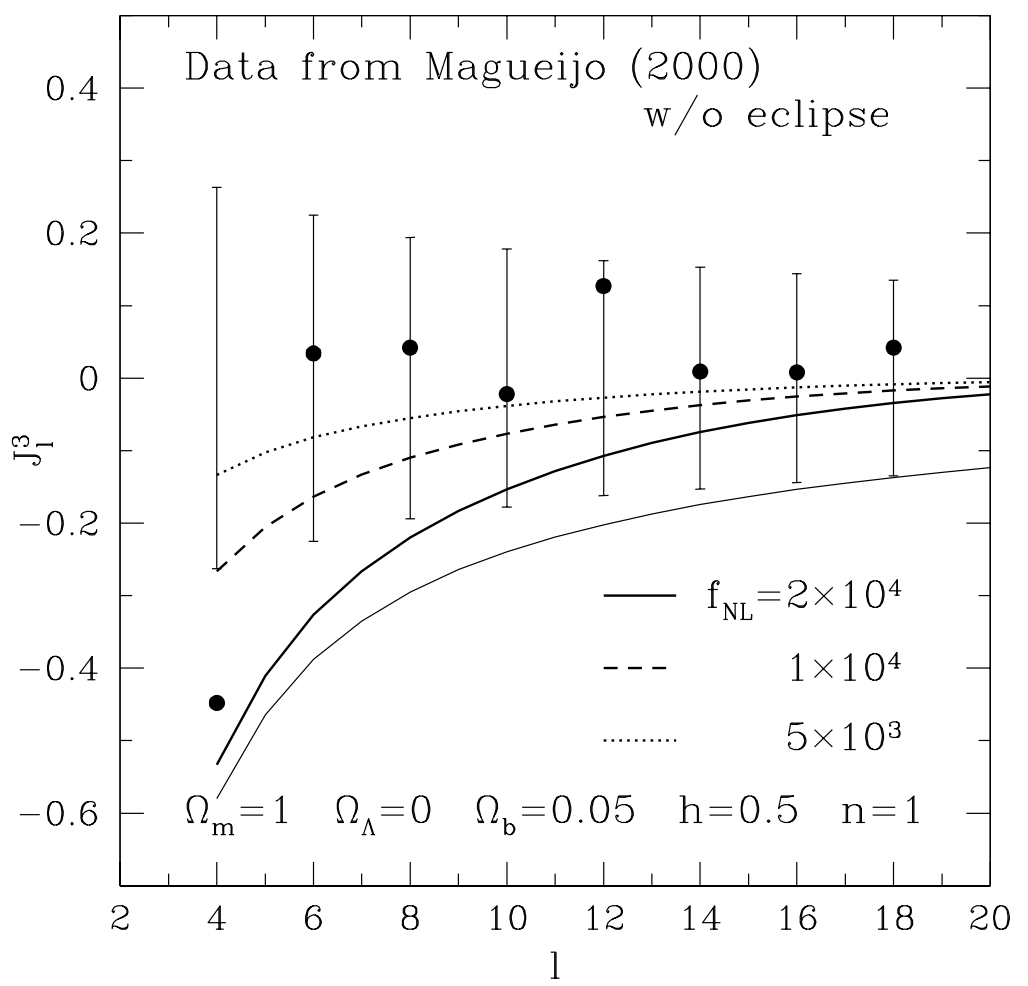

Figure 2. The comparison of theoretical primary bispectrum to the COBE 4 year data, $J_{l}^{3}$ (Eq.(14)), defined by Magueijo (2000). Filled circles are measured $J_{l}^{3}$, while thick solid, dashed, and dotted lines correspond to the primary bispectrum with $f_{N L}=2 \times 10^{4}, 10^{4}$, and $5 \times 10^{3}$, respectively. Error bars are rms scatters from the Gaussian Monte Carlo realizations. Note that the beam-smearing effect has been taken into account. The thin solid line shows the un-smoothed case for $f_{N L}=2 \times 10^{4}$.

8. U. Seljak and M. Zaldarriaga, Astrophys. J. 469, 437 (1996).

9. E. Komatsu and D. N. Spergel, Phys. Rev. D (submitted); preprint, astro$\mathrm{ph} / 0005046$.

10. J. Magueijo, Astrophys. J. Lett. 528, L57 (2000).

11. D. M. Goldberg and D. N. Spergel, Phys. Rev. D 59, 103002 (1999).

12. A. Cooray and W. Hu, Astrophys. J. 534, 533 (2000).

13. A. Refregier, D. N. Spergel and T. Herbig, Astrophys. J. 531, 31 (2000).

14. A. Refregier, E. Komatsu, D. N. Spergel and U.-L. Pen, Phys. Rev. D 61, 123001 (2000).

15. Ya. B. Zel'dovich and R. A. Sunyaev, Astrophys. Space. Sci. 4, 301 (1969).

16. D. N. Spergel and D. M. Goldberg, Phys. Rev. D 59, 103001 (1999).

17. A. Gangui and J. Martin, Mon. Not. R. Astron. Soc., 313, 323 (2000); Phys. Rev. D 62, 103004 (2000). 
18. P. G. Ferreira, J. Magueijo and K. M. Górski, Astrophys. J. Lett. 503, L1 (1998).

19. A. J. Banday, S. Zaroubi and K. M. Górski, Astrophys. J. 533, 575 (2000).

bispectrum mg9 long: submitted to World Scientific on October 24, 2018 